\title{
The symmetry energy, neutron skin thickness and isovector dipole response of neutron-rich nuclei
}

\author{
A. $\operatorname{HoRvat}^{1 *}$, N. $\operatorname{PAAR}^{2}$ \\ ${ }^{1}$ Institut für Kernphysik, TU Darmstadt, Germany \\ ${ }^{2}$ Department of Physics, Faculty of Science, University of Zagreb, Croatia
}

\begin{abstract}
The isotopic evolution of the relationship between the symmetry energy at saturation density of nuclear matter $(J)$, neutron skin thickness $(\Delta R)$ and relevant observables related to isovector dipole excitations in neutron rich ${ }^{116-136} \mathrm{Sn}$ isotopes has been investigated in the framework of relativistic nuclear energy density functional theory. The description employs a family of effective interactions with density dependent meson-nucleon couplings (DDME) spanning the range of values $J=30-38 \mathrm{MeV}$.
\end{abstract}

\section{Introduction}

Collective excitations in neutron rich nuclei contain valuable information which allow to investigate the properties of symmetry energy of the nuclear equation of state. The knowledge on the symmetry energy and its density dependence is of particular importance for understanding a variety of phenomena in finite nuclei as well as in neutron stars. Using relativistic nuclear energy density functional (EDF) in describing both the nuclear matter properties as well as collective excitations in finite nuclei, a direct link can be established between the symmetry energy at saturation density $(J)$, the neutron skin thickness $(\Delta R)$ and observables related to nuclear collective motion in neutron rich nuclei.

The neutron skin thickness represents a ground state property of finite nucleus that is an indicator of the isovector properties of effective nuclear interactions. It is linearly correlated with the symmetry energy parameters

\footnotetext{
*For the R3B-LAND Collaboration
} 


\section{EPJ Web of Conferences}

at the saturation density, but also very difficult to measure in a model independent way. For a set of chosen EDFs consistent with the measured value of the dipole polarizability $\left(\alpha_{D}\right)$ in ${ }^{208} \mathrm{~Pb}$ [1], a systematic variation of parameters of the phenomenological interactions reveals a strong correlation between $\alpha_{D}$ and $\Delta R$ within a family of effective interactions, serving as a constraint on the value of $\Delta R$ in conjuncture with the measured $\alpha_{D}$ [2]. Furthermore, an even better correlation was found between $\alpha_{D} J$ and the slope of the symmetry energy $L$, and therefore $\Delta R$, which holds across different sets of EDFs, as indicated by the droplet model [3].

A statistical covariance analysis for the DDME-min1 effective interaction was used to explore correlations between various strength related E1 observables and $J, L$ and $\Delta R$ [4]. In contrast to similar analyses using Skyrme functionals [5], it showed relevant correlations for the low lying isovector pygmy dipole strength (PDS). In the present study, the sensitivities of these observables towards variations of $J$ and $\Delta R$ are investigated by employing a family of DD-ME effective interactions spanning a range of values $J=30-38$ $\mathrm{MeV}$ [6]. Calculations are carried out by using the framework of relativistic Hartree-Bogoliubov (RHB) model and relativistic quasiparticle random phase approximation (RQRPA).

\section{Results}

The dipole response of a nucleus to a time varying external excitation is described in terms of reduced transition probabilities. Figure 1 shows the transition strength distribution for ${ }^{132} \mathrm{Sn}$ using the isovector dipole transition operator and DDME effective interactions. The strength distribution is characterized by its moments

$$
m_{k}(T, J)=\sum_{\nu} E_{\nu}^{k} B^{T}\left(J, E_{\nu}\right),
$$

where the sum can be capped at a certain energy in order to consider only the PDS contributions. The $k=0$ moment is the total strength, while $m_{-1}$ is proportional to the dipole polarizability $\alpha_{D}$, on which the pygmy states have a significant influence because of inverse energy weighing. With regards to the recent measurements of the E1 response of the neutron rich side of the Sn isotope chain carried out using the LAND-R3B setup at GSI in Darmstadt, for which the analysis in under way, it is useful to examine how sensitive these observables are to variations in the symmetry energy in a systematic way. 


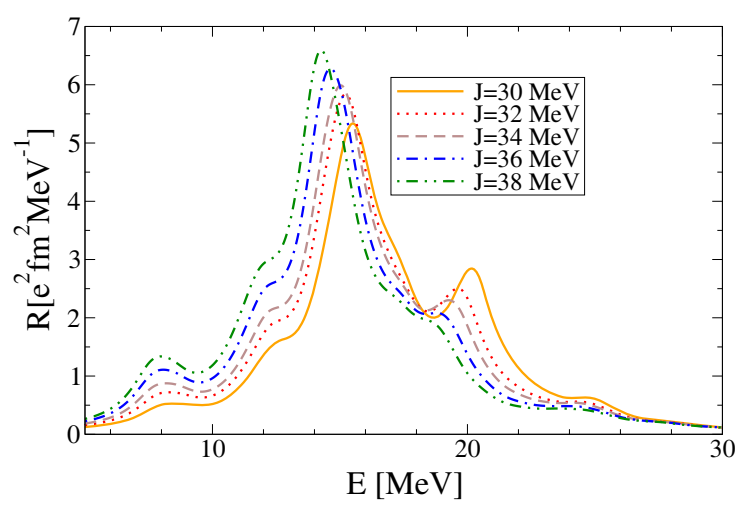

Figure 1: The isovector E1 response of ${ }^{132} \mathrm{Sn}$ using the DD-ME family of effective interactions spanning the values of $J$ denoted in the legend.

Figure 2 shows $\alpha_{D}$ and $m_{0}$ as functions of $J$ and $\Delta R$. On the right hand side the sum for these quantities was capped at $10 \mathrm{MeV}$ to consider only the PDS part. The dipole polarizability is found to be more sensitive than $m_{0}$, since the increase of the total strength and decrease of the giant resonance excitation energy both increase the total $\alpha_{D}$. The slope of both observables becomes much steeper for the low lying part of the spectra, as observed in a study using the FSUGold and NL3 families of the effective interactions [7].
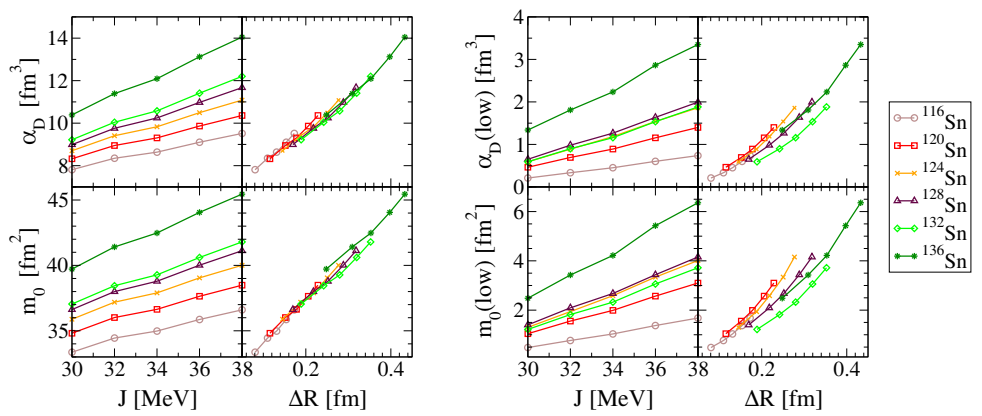

Figure 2: Dipole polarizability and the transition strength for ${ }^{116-136} \mathrm{Sn}$ isotope chain calculated for the entire excitation spectra (left) and the low-lying states (right) displayed as functions of $\mathrm{J}$ and $\Delta R$.

When constrained to the low energy states only, both observables have a similar slope for a given isotope, since the energy at which these states appear displays weak sensitivity to variations in $J$ (see Fig. 1). More neutron rich isotopes appear to exhibit a stronger sensitivity to variations in 
$J$, however, if viewed with $\Delta R$ as a variable, the sensitivity decreases with neutron number towards the neutron rich ${ }^{132} \mathrm{Sn}$. The reason for this is that the slope of $\Delta R$, when viewed as a function of $J$, displays a steeper slope for more neutron rich isotopes, similar as within the droplet model.

\section{Conclusion}

Valuable information on nuclear matter properties related to isospin asymmetry can be obtained from experimental data on the electric dipole response of neutron rich nuclei [8]. Within the relativistic EDF framework, E1 strength related observables display a greater sensitivity to the value of the symmetry energy at saturation density if constrained to the PDS states.

\section{Acknowledgments}

A. Horvat would like to thank HGS-HIRe for travel support.

\section{References}

[1] A. Taami et al., Phys. Rev. Lett. 107, 062502 (2011).

[2] J. Piekarewicz et al., Phys. Rev. C 85 041302(R) (2012).

[3] X. Roca-Maza et al., Phys. Rev. C., 88, 024316 (2013).

[4] N. Paar, A. Horvat, EPJ Web of Conferences 66, 02078 (2014).

[5] P.-G. Reinhard and W. Nazarewicz, Phys. Rev. C 81, 051303 (2010).

[6] D. Vretenar, T. Nikšić and P. Ring, Phys. Rev. C 68, 024310 (2003).

[7] J. Piekarewicz, Phys. Rev. C 83, 034319 (2011).

[8] D.M. Rossi et al., Phys. Rev. Lett. 111, 242503 (2013). 